\title{
Interstellar dust close to the Sun
}

\author{
Priscilla C. Frisch ${ }^{1}$ and Jonathan D. Slavin ${ }^{2}$ \\ ${ }^{1}$ Dept. Astronomy and Astrophysics, University of Chicago \\ ${ }^{2}$ Harvard-Smithsonian Center for Astrophysics
}

(Received February 13, 2012; Revised May 2, 2012; Accepted May 2, 2012; Online published March 12, 2013)

\begin{abstract}
The low density interstellar medium (ISM) close to the Sun and inside of the heliosphere provides a unique laboratory for studying interstellar dust grains. Grain characteristics in the nearby ISM are obtained from observations of interstellar gas and dust inside of the heliosphere and the interstellar gas towards nearby stars. Comparison between the gas composition and solar abundances suggests that grains are dominated by olivines and possibly some form of iron oxide. Measurements of the interstellar $\mathrm{Ne} / \mathrm{O}$ ratio by the Interstellar Boundary Explorer spacecraft indicate that a high fraction of interstellar oxygen in the ISM must be depleted onto dust grains. Local interstellar abundances are consistent with grain destruction in $\sim 150 \mathrm{~km} \mathrm{~s}^{-1}$ interstellar shocks, provided that the carbonaceous component is hydrogenated amorphous carbon and carbon abundances are correct. Variations in relative abundances of refractories in gas suggest variations in the history of grain destruction in nearby ISM. The large observed grains, $>1 \mu \mathrm{m}$, may indicate a nearby reservoir of denser ISM. Theoretical three-dimensional models of the interaction between interstellar dust grains and the solar wind predict that plumes of $\sim 0.18 \mu \mathrm{m}$ dust grains form around the heliosphere.
\end{abstract}

Key words: Interstellar, dust, heliosphere, abundances.

\section{Introduction}

The local interstellar medium (ISM) surrounding the Sun and inside of the heliosphere offers a unique set of diagnostics for interstellar dust grains, in a well-defined region of space. In the local low density ISM, interstellar dust grains (ISDGs) can be studied far from the dense clouds where grain mantle accretion occurs. Starlight reddening data show that the ISM opacity becomes significant only beyond $\sim 70-100$ pc (Fitzgerald, 1968). Because of the low densities of the Local Bubble, $n<0.005 \mathrm{~cm}^{-3}$, interstellar shocks propagate relatively long distances before dissipating. Local clouds are partially ionized and have low column densities which lead to the need to include ionization corrections to calculate the gas phase abundances of refractory elements and thereby infer the composition of the dust (Frisch et al., 2011). Variations in the relative abundances of $\mathrm{Fe}, \mathrm{Mg}$, and $\mathrm{Si}$ in the local ISM indicate variations in the processing history of the dust over parsec-sized spatial scales. In the absence of infrared-bright dense clouds, the composition of local dust is inferred from measurements of interstellar gas towards nearby stars and inside of the heliosphere. In situ measurements of neutral interstellar gas and dust grains provide a benchmark for understanding the Local Interstellar Cloud (LIC) surrounding the heliosphere (Frisch et al., 2009; Bochsler et al., 2012). The mass distribution is given by in situ observations of interstellar dust in the heliosphere, at least for the larger grains. A unique local diagnostic of the gas-to-dust mass ratio, $R_{\mathrm{g} / \mathrm{d}}$, is provided

Copyright (c) The Society of Geomagnetism and Earth, Planetary and Space Sciences (SGEPSS); The Seismological Society of Japan; The Volcanological Society of Japan; The Geodetic Society of Japan; The Japanese Society for Planetary Sciences; TERRAPUB.

doi:10.5047/eps.2012.05.001 by comparisons between in situ dust data and the local ISM properties inferred from observations of interstellar gas inside and outside of the heliosphere. The observed deficit of small grains is attributed to the interaction of ISDGs with the heliosphere, while the large-grain excess provides fundamental information about the origin and destruction of grains in the ambient ISM.

\section{Characteristics of Local Interstellar Medium}

The local ISM is clumped into low density clouds that flow past the Sun with a mean bulk velocity of $17 \mathrm{~km} \mathrm{~s}^{-1}$ in the local standard of rest (LSR), and away from the direction of the center of the Loop I superbubble near $\ell=335^{\circ}$, $b=-5^{\circ}$. The low local densities, $\left\langle n_{\mathrm{HI}}\right\rangle \sim 0.1 \mathrm{~cm}^{-3}$ and $N\left(\mathrm{H}^{\mathrm{o}}\right)<10^{18.5} \mathrm{~cm}^{-2}$, correspond to extinctions of $\mathrm{E}(\mathrm{B}-\mathrm{V})$ $<0.001$ mag towards nearby stars. Interstellar dust within $\sim 15 \mathrm{pc}$ of the Sun is the topic of this discussion. Dust properties can be inferred from observations of gas towards stars within $\sim 40 \mathrm{pc}$, since most nearby ISM within $40 \mathrm{pc}$ is also within 15 parsecs, and also from in situ observations of interstellar dust inside the heliosphere. Local clouds are traced by optical and UV absorption lines, and sorted into clouds based on common velocities (Fig. 1). For a recent review of local ISM properties see Frisch et al. (2011).

Over the past 15 Myrs, stellar winds and supernova from the nearby stellar associations in Scorpius, Centaurus, and Orion have formed superbubbles extending to the solar vicinity (e.g. Loop I and the Orion superbubble). Loop I is a giant $\left(\sim 90^{\circ}\right.$ radius) radio loop of polarized synchrotron emission located in the northern hemisphere, and surrounding the Scorpius-Centaurus Association. The superbubble shell is about 4 Myrs old (Frisch, 1996). Models of Loop I that assume it is a spherical object place the Sun in or 


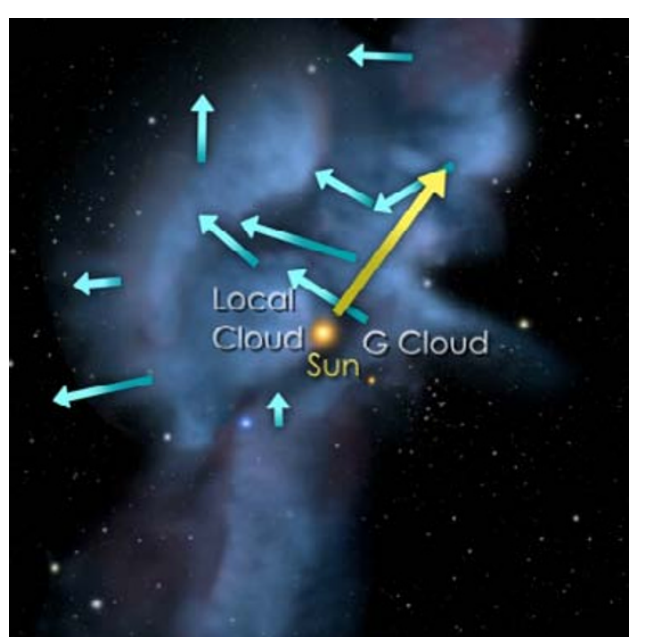

Fig. 1. Parts of the cluster of local interstellar clouds within $10 \mathrm{pc}$ are shown projected onto the galactic plane. The yellow dot shows the location of the Sun and the yellow arrow gives the direction of solar motion through the LSR. Cloud motions relative to the LSR are shown with blue arrows. The galactic center is towards the right of the figure, and $\ell=90^{\circ}$ is at the figure top. The bulk motion of these clouds is away from the center of the Loop I superbubble. For more information on the cloud names and velocities see figure 11 in Frisch et al. (2011) and Redfield and Linsky (2008). (Figure credit: Adler Planetarium, NASA, Priscilla Frisch, Seth Redfield, Jeff Linsky)

near the shell rim. The bulk motion of the cluster of local interstellar clouds (CLIC) is flowing from a position near the center of Loop I, as defined by the $\mathrm{S} 1$ superbubble shell centered $78 \pm 10 \mathrm{pc}$ away at $\ell=346^{\circ} \pm 5^{\circ}, b=-3^{\circ} \pm 5^{\circ}$ (Wolleben, 2007; Frisch et al., 2012).

Both the kinematics of the CLIC and the direction of the local magnetic field suggest that the CLIC is associated with an evolved superbubble shell. The local field direction has been derived both from the weak polarizations of nearby stars, $0.0001 \%-0.02 \%$ (Frisch et al., 2010, 2012), and from the interstellar magnetic field direction defined by the "ribbon" of interstellar neutral atoms observed by the Interstellar Boundary Explorer (IBEX) spacecraft (McComas et al., 2009). These data indicate that the ISMF within several parsecs is approximately parallel to the rim (or perpendicular to a surface normal) of the S1 shell (Frisch et al., 2010, 2012). Together these data suggest that interstellar dust grains within several parsecs have recently been subjected to interstellar shocks.

\section{Composition of Local Interstellar Dust}

In the low density local ISM, where dust emission is difficult to observe, the interstellar dust composition is inferred from the assumption that atoms missing from the gas-phase are depleted onto dust grains. This method requires knowledge of the chemical composition of the ISM. The isotopic composition of the LIC that feeds ISM into the heliosphere indicates that the reference abundances for the LIC may be solar. LIC neutrals are ionized inside of the heliosphere, forming pickup ions that are accelerated to become the anomalous cosmic rays (energy $<70$ $\mathrm{MeV} /$ nucleon). Anomalous cosmic rays have isotopic compositions that are approximately solar, ${ }^{20} \mathrm{Ne} /{ }^{22} \mathrm{Ne} \sim 13.7$ and ${ }^{16} \mathrm{O} /{ }^{18} \mathrm{O} \sim 500$, instead of showing galactic cosmic ray ratios (Leske, 2000). X-ray spectroscopy of absorption edges in X-ray binaries also show ratios of interstellar $\mathrm{Ne} / \mathrm{O}$ that are consistent with the solar value (Juett et al., 2006).

Three means of determining the composition of local dust grains are discussed, based on: (1) refractory elements that are missing from the gas-phase; (2) photoionization models of the interstellar gas entering the heliosphere, that reconstruct the depletion pattern of the gas; (3) in situ detections of interstellar $\mathrm{Ne}$ and $\mathrm{O}$ in the heliosphere. Methods 2 and 3 both indicate that a large fraction $(35 \%-40 \%)$ of the oxygen in the local ISM must be depleted onto dust grains.

\subsection{Refractory elements in CLIC}

Surveys of interstellar absorption lines towards nearby stars that sample the CLIC (e.g. Redfield and Linsky, 2002, 2004; and references therein) have shown that the relative gas-phase abundances of Fe and $\mathrm{Mg}$ vary within $15 \mathrm{pc}$ of the Sun (Fig. 2, left). A fit to the gas-phase abundances of $\mathrm{Mg}$ vrs Fe suggests that $\mathrm{N}\left(\mathrm{Fe}^{+}\right) \propto \mathrm{N}\left(\mathrm{Mg}^{+}\right)^{0.57}$, which indicates that grain destruction returns more $\mathrm{Mg}$ than Fe to the gas. Although $\mathrm{H}$ ionization varies by up to $66 \%$ in the nearby ISM, Fe, Mg, and Si are mainly singly ionized (Section 3.2, Slavin and Frisch, 2008), so that $\mathrm{Fe}^{+} / \mathrm{Mg}^{+}$ratio is relatively insensitive to ionization variations. This scenario suggests the presence of additional iron-rich grains in the local ISM, with iron oxides a likely possibility (Jones, 1990). Typical depletion patterns of Fe, Mg, and Si shown in Fig. 2, right, also suggest the presence of at least two grain components, one consistent with amorphous olivines $\left(\mathrm{MgFeSiO}_{4}\right)$ where $\left(\mathrm{Mg}_{\text {dust }}+\mathrm{Fe}_{\text {dust }}\right) / \mathrm{Si}_{\text {dust }} \approx 2$, and an additional iron-rich component in the dust. A third characteristic of the depletion pattern appears when depletions in tenuous and translucent clouds are combined to display the gas-to-dust mass ratio, $R_{\mathrm{g} / \mathrm{d}}$, as a function of the percentage of the dust mass carried by Fe (Fig. 3, left, and Frisch and Slavin (2003)). The increase of $R_{\mathrm{g} / \mathrm{d}}$ with Fe-dominance of the core indicates that grain destruction preferentially destroys lighter grain mantle material and leave behind ironrich cores (also see Section 5.1). Consistent with these characteristics are that gas-phase interstellar $\mathrm{Mg}$ abundances are found to scale with Si abundances, and sometimes independently of $\mathrm{Fe}$ abundances, both in low density galactic sightlines and in the Small Magellenic Cloud, indicating that there is a separate Fe-rich grain component (Howk et al., 1999; Welty et al., 2001; Cartledge et al., 2006; Sofia et al., 2006). Frisch et al. (1999) found that the composition of local grains are consistent with olivine cores and pyroxene mantles.

Kimura et al. (2003a) looked in detail at the local grain mineralogy, using the assumption that the local interstellar cloud is $30 \%$ ionized. They found that the grain mineralogy is quite sensitive to the assumed solar abundances. By comparing depletion patterns with likely grain models and the condensation sequence of minerals, a viable model of a LIC grain contains an Mg-rich olivine and pyroxene core with Fe-rich kamacite, and mantles composed of organic refractory compounds of photoprocessed $\mathrm{C}, \mathrm{H}, \mathrm{O}$ and $\mathrm{N}$ ices. For this scenario, $\sim 45 \%$ of the oxygen is depleted primarily into organic refractory mantles $(\mathrm{CHON})$ and enstatite $\left(\mathrm{MgSiO}_{3}\right)$, and the grain composition is found to be consistent with grains in molecular clouds. 

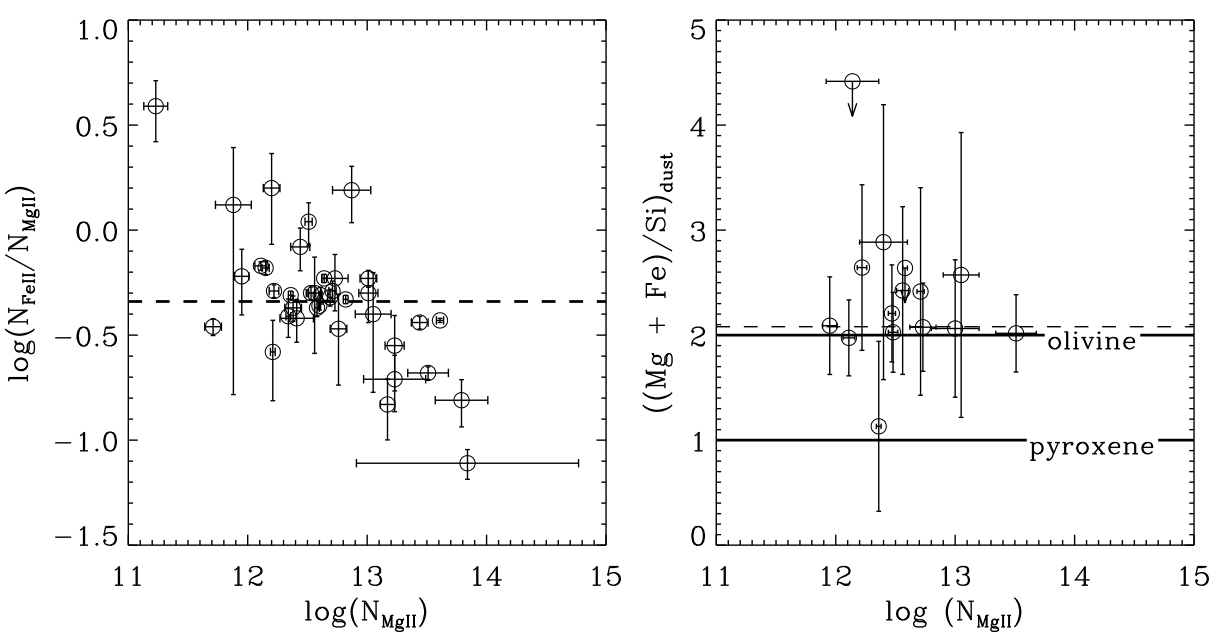

Fig. 2. The observed gas-phase column densities of $\mathrm{Mg}^{+}, \mathrm{Fe}^{+}$, and $\mathrm{Si}^{+}$in the local ISM indicate that although there is a wide variation in the relative amount of $\mathrm{Fe}^{+}$when compared to $\mathrm{Mg}^{+}$(left), the primary dust component has $(\mathrm{Mg}+\mathrm{Fe}) / \mathrm{Si} \sim 2$ such as expected for olivine silicates (right). The comparable value for pyroxene is $(\mathrm{Mg}+\mathrm{Fe}) / \mathrm{Si} \sim 1$. Grain destruction returns more $\mathrm{Mg}$ than $\mathrm{Fe}$ to the gas-phase. The dashed line in the left figure shows the ratio predicted by photoionization models of the LIC (Slavin and Frisch, 2008), where it is also predicted that $15 \%$ of the Mg is doubly ionized (Slavin and Frisch, 2008). The dust composition on the right is obtained by comparing the gas-phase abundances of the elements with solar abundances. The presence of iron oxides in the dust would help explain the highest ratios (This figure is courtesy of Seth Redfield, using results from Redfield and Linsky (2002, 2004) and Frisch et al. (2011).).

(a)

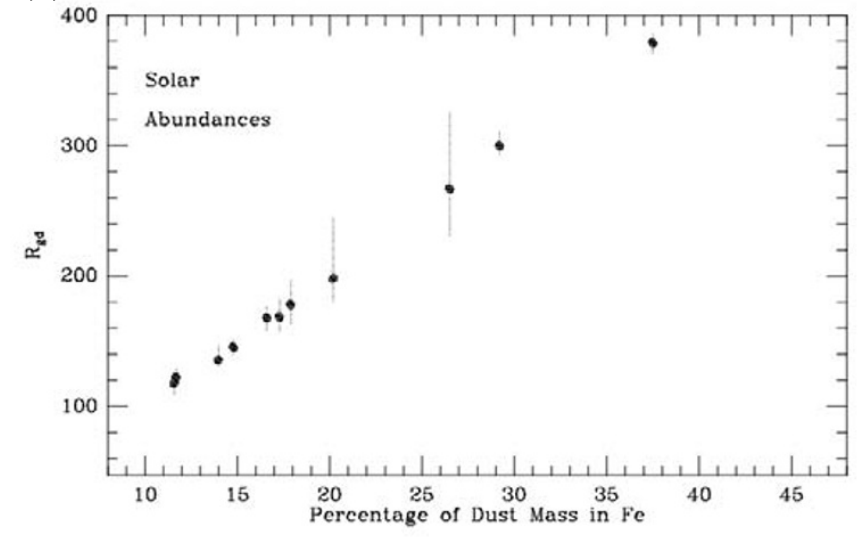

(b)

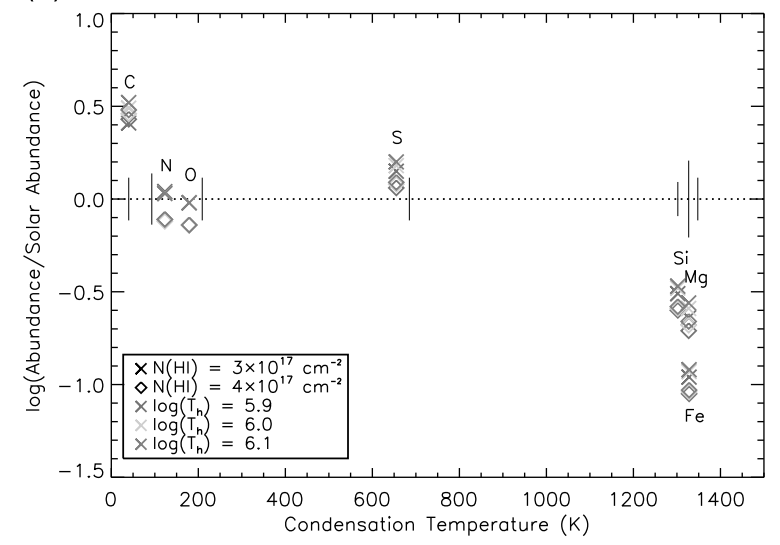

Fig. 3. Left: Percentage of dust mass carried by Fe as a function ol the gas-to-dust mass ratio $R_{\mathrm{g} / \mathrm{d}}$ (from Frisch and Slavin, 2003). The interstellar column densities for these stars span three orders of magnitude, with the smallest values of $R_{\mathrm{g} / \mathrm{d}}$ corresponding to the lowest percentages of Fe in the grain mass. Right: Abundances relative to Asplund et al. (2005) solar abundances vs. condensation temperature. Results from models of the ionization for the $\epsilon$ CMa line of sight including radiative transfer (Model 26 in Slavin and Frisch (2008)). The symbol shape indicates the assumed H I column density for the model, while the color indicates the assumed temperature for the hot gas of the Local Bubble. The abundances are fixed so as to match the observed ion column densities. An overabundance of carbon in the gas phase is required to match the observed carbon abundances, suggesting that carbonaceous dust grains have been destroyed in the LIC. The carbon abundance in these models has been reconstructed from the cloud ionization and the $1335.7 \AA$ carbon fine-struture line (see Slavin and Frisch, 2008 for additional information).

\subsection{Elemental depletion patterns for interstellar gas entering heliosphere}

A unique aspect of the ISM enveloping the heliosphere is that the cloud properties can be derived from in situ data as well as sightline-integrated observations of nearby stars. Measurements of interstellar $\mathrm{He}^{\circ}$ and other interstellar neutrals inside of the heliosphere sample the ionizations of the gas entering the heliosphere, while measurements of the LIC towards nearby stars gives a complete picture of sightline-integrated ionization. Photoionization models of the LIC therefore reconstruct the full gas-phase abundances of refractory elements, which are predominantly singly ionized in the local ISM.

Model 26 in Slavin and Frisch (2008) provides a viable model of the depletion patterns of the ISM seen inside and outside of the heliosphere (Fig. 3). In this model hydrogen is $22 \%$ ionized, helium is $39 \%$ ionized, and $15 \%$ of the magnesium and neon are doubly ionized. These ionization levels show that full ionization models are required to reconstruct the depletion pattern for low density gas. For this model, oxygen has a total (ions plus neutrals) gas phase abundance of $331 \mathrm{ppm}$, so that $35 \%$ of the oxygen is depleted onto dust grains. The He ionization is provided by radiation generated in a conductive interface at the boundary of the LIC and surrounding hot gas, as well as starlight from nearby white dwarfs. The depletion pattern in the LIC is consistent with grains consisting of amorphous olivine. These photoionization models yield a supersolar 
carbon abundance in the LIC gas at the heliosphere, leaving no carbon for the dust (Fig. 3) and indicating that the carbonaceous grains have been destroyed in the LIC (Section $5)$. Reconstruction of the gas-phase abundances in the LIC gives a value for the gas-to-dust mass ratio, $R_{\mathrm{g} / \mathrm{d}}$, that explicitly includes hidden ionized gas. These models predict values for $R_{\mathrm{g} / \mathrm{d}}$ that are consistent with in situ measurements (see below).

\subsection{Dust composition from IBEX measurements of in-} terstellar neon and oxygen

A new diagnostic of the oxygen composition of ISDGs has recently been provided by in situ measurements of interstellar $\mathrm{Ne}^{\mathrm{o}}$ and $\mathrm{O}^{\circ}$ by the Interstellar Boundary Explorer (IBEX) mission (Bochsler et al, 2012). Interstellar $\mathrm{Ne}^{\mathrm{o}}$ and $\mathrm{O}^{\circ}$ flow into the inner heliosphere from the upwind direction, near the galactic center direction, where a special observing configuration allows the neutral flow to be measured by the IBEX-LO instrument. From these data an interstellar ratio of $(\mathrm{Ne} / \mathrm{O})=0.27 \pm 0.10$ is obtained. In order to obtain this value, the measured particle fluxes are corrected for propagation through the heliosphere, ionization of the neutrals in the heliosphere and heliosheath regions (the latter termed "filtration"), and the ionizations of $\mathrm{Ne}$ and $\mathrm{O}$ in the ISM surrounding the heliosphere (which are provided by photoionization models of the LIC (Slavin and Frisch, 2008)). The solar ratio is $(\mathrm{Ne} / \mathrm{O}) \sim 0.17$. Since $\mathrm{Ne}$ will be undepleted in the ISM, the IBEX $\mathrm{Ne} / \mathrm{O}$ ratio therefore indicates that $\sim 40 \%$ of the interstellar oxygen in the ISM surrounding the heliosphere has been depleted onto interstellar dust grains.

Both the photoionization models and in situ $\mathrm{Ne} / \mathrm{O}$ data indicate that $35 \%-40 \%$ of the oxygen in the LIC is missing from the gas phase and therefore depleted onto dust grains. This pattern is consistent with the global depletion pattern of oxygen in the ISM. Jenkins (2009) has found that oxygen depletes more rapidly then refractories such as $\mathrm{Mg}, \mathrm{Si}$ and $\mathrm{Fe}$ for all levels of depletions. In the low density local ISM, it is difficult to identify the compounds that lock up so many oxygen atoms in a solid phase since water ices are not observed in high abundances. Jenkins argues that water ices may coat grains larger than $1 \mu \mathrm{m}$ that are difficult to detect. Pure metallic iron is unlikely in the ISM because of rapid oxidation, but iron oxides are a possible hidden reservoir for oxygen (Jones, 1990). The missing O may be in organic refractory grains such as seen by Stardust (Kimura et al., 2003a; Whittet, 2010). Whittet (2010) argue that the oxidation ratios of metals in dust would correspond to $\mathrm{O} /(\mathrm{Mg}+\mathrm{Si}+\mathrm{Fe})=1.5$ for a grain population of $\mathrm{MgSiO}_{3}$ silicates and $\mathrm{Fe}_{2} \mathrm{O}_{3}$ oxides, and 1.2 for a mixture of $\mathrm{FeO}$ and $\mathrm{MgO}$ monoxides and primarily olivine silicates. For the LIC, where the level of ionization in the gas is reconstructed with photoionization models, an oxidation ratio of 1.8 is found from model 26 in Slavin and Frisch (2008) using abundances from Asplund et al. (2009). Observations of six clouds towards four nearby stars within $32 \mathrm{pc}$ give a mean oxidation ratio of $2.5 \pm 0.7$.

The process of inferring the oxygen budget in the ISM is subject to several major uncertainties, including the correct reference abundance in the ISM and the composition of grains surviving shock destruction. Based on the iso- topic composition of the anomalous cosmic ray component, the ISM flowing into the heliosphere has a solar composition (Section 3). The solar oxygen abundance is $490 \pm 58$ ppm, which is twice the oxygen abundance in meteorites (Asplund et al., 2009). Cartledge et al. (2006) concluded that the problem with "missing oxygen" is minimized for an assumed reference standard for the ISM of young F and G-star abundances, and a grain population of silicates and $\mathrm{FeO}$ oxides.

\section{Interstellar Grains in the Heliosphere}

Interstellar dust grains have been measured within 5 AU of the Sun by spacecraft (Mann, 2009). Interstellar gas and dust flowing into the heliosphere have the same velocity vectors when extrapolated to the ISM at "infinity". Ulysses data give a dust velocity at infinity of $24.5_{-1.2}^{+1.1} \mathrm{~km} \mathrm{~s}^{-1}$ (Kimura et al., 2003b) towards galactic coordinates $\ell, b=188^{\circ} \pm 15^{\circ},-14^{\circ} \pm 4^{\circ}$ (Frisch et al., 1999). The gas velocity at infinity, based on the concensus IBEX-LO observations of interstellar $\mathrm{He}^{\circ}$ in the inner heliosphere, is $23.2 \pm 0.3 \mathrm{~km} \mathrm{~s}^{-1}$ towards the direction $\ell, b=185.25^{\circ} \pm 0.24^{\circ},-12.03^{\circ} \pm 0.51^{\circ}$, with a temperature of $6,300 \pm 390 \mathrm{~K}$ (McComas et al., 2012). A bow shock around the heliosphere partially decouples the gas and dust components of the ISM, so the dynamical interaction of small grains with the heliosphere, in particular, is sensitive to the presence of a bow shock. For the new IBEXLO LIC velocity vector, the heliosphere will not have a bow shock for magnetic field strengths $>3 \mu \mathrm{G}$.

In principle, if the propagation of interstellar dust grains through the heliosphere is known, the grain mass distribution (for the larger grains) in the ISM can be recovered. A nominal power-law MRN distribution (Mathis et al., 1977) is assumed for this comparison. Although the MRN is not a realistic description of the extinction and emission properties of interstellar dust over the full spectral range from the infrared to ultraviolet, it plays a key role in the interpretation of astronomical dust data (Zubko et al., 2004). In the absence of reliable data on the mass distribution of the dust in the ISM outside of the heliosphere, the heliospheric modulation of interstellar dust is often discussed in terms of the MRN distribution. (We note that the calculations of grain trajectories do not depend on the size distribution.) A distinct property of the mass distribution of interstellar grains in the heliosphere is the deficit of small grains, mass $<10^{-15}$ gr (radius $0.04-0.2 \mu \mathrm{m}$ for density $2.5 \mathrm{gr} \mathrm{cm}^{-3}$ ), and an excess of large grains, masses $>10^{-10} \mathrm{gr}$. The deficit is caused by the deflection of the small grains around the heliosphere (Frisch et al., 1999; Slavin et al., 2010, 2011), while the excess of large grains, radii $>1 \mu \mathrm{m}$ for a nominal density of $2.5 \mathrm{~cm}^{-3}$, signals an unexpected component of local interstellar dust. In 2005/2006 the dust flow direction shifted southwards by $\sim 30^{\circ}$, probably due to the change in the solar wind magnetic polarity (Krüger et al., 2010).

The gas-to-dust mass ratio, $R_{\mathrm{g} / \mathrm{d}}$, can be independently determined from the in situ dust measurements, and then compared with the predictions of the LIC photoionization models. A value of $R_{\mathrm{g} / \mathrm{d}} \sim 150$ is consistent both with recent Ulysses data (H. Krueger, private communication) and the photoionization models (which give a range of $R_{\mathrm{g} / \mathrm{d}}$ 
$=140-320$, depending on the selected solar abundance (Slavin and Frisch, 2008)). The in situ value for $R_{\mathrm{g} / \mathrm{d}}$ only depends on the photoionization models for the total gas mass, and otherwise this $R_{\mathrm{g} / \mathrm{d}}$ should be an upper limit because small grains are excluded from the heliosphere. If the values for $R_{\mathrm{g} / \mathrm{d}}$ determined separately from the dust measurements and observations of the gas (combined with the missing-mass argument) do not agree, it would indicate that the grains and gas are from different reservoirs, which is to say a decoupling of gas and dust leading to a local dust enhancement. First indications are that $R_{\mathrm{g} / \mathrm{d}}$ in the LIC as derived from the in situ dust measurements will decrease significantly once the mass of the excluded grains is included.

Interstellar dust grains encountering the heliosphere will interact most strongly with the magnetic field. The trajectories resulting from this interaction can be quite complex, depending on the charge-to-mass ratio for the grain and the detailed morphology of the magnetic field throughout the heliosphere. The grains are generally charged positively because of the strength of the solar UV/optical radiation field which produces photoejection of electrons from the grains. We have used numerical magneto-hydrodynamical (MHD) + kinetic (for the neutrals) calculations of the heliosphere to study the trajectories of interstellar grains incident on the heliosphere (Slavin et al., 2010, 2011, 2012). We used two different solar wind magnetic field (SWMF) polarities in our modeling, a de-focusing configuration (south pole positive, corresponding to the current polarity) and focusing polarity (north pole positive).

Interstellar dust enters the heliosphere region at the relative speed of the solar system and the LIC. The smallest grains $(\sim 0.01 \mu \mathrm{m})$ which have gyroradii that are small on the scale of the heliosphere $(\leq 1 \mathrm{AU})$ couple tightly to the interstellar field and their trajectories follow that field as it is distorted by the heliosphere. Somewhat larger grains $(\sim 0.1$ $\mu \mathrm{m}$ ) have gyroradii large enough to allow them to penetrate the heliopause, at which point their trajectories depend on the details, in particular the polarity, of the SWMF. If the field has magnetic north coinciding with the Sun's north pole, the grains are deflected away from the ecliptic while for the opposite polarity, they are focused in the ecliptic plane. For intermediate sized grains, $\sim 0.05-0.2 \mu \mathrm{m}$, the SWMF polarity has strong effects on the grain trajectories and resulting grain density, either concentrating the grains near the north and south ecliptic poles (de-focusing polarity) or in the ecliptic plane (focusing polarity). Figure 4 shows a 3-D rendering of the dust density distribution for $0.18 \mu \mathrm{m}$ grains. For grains that are even larger, their gyroradii can be $\sim 1000 \mathrm{AU}$ or larger and tend to be only slightly deflected from their initial trajectory. For these grains the effect of the Sun's gravity leads to an enhanced space density in the inner solar system.

The very different density distributions of the grains in the models that use different SWMF polarities indicate that we need to take into account the time evolution of the heliosphere in such calculations. An interstellar grain will take $\sim 30$ yr to go from the heliopause to the Sun and so will experience two polarity changes because of the solar cycle. The SWMF polarity of the 1990's was de-focusing, while that of the 2000's was focusing. By calculating the enhancement in grain space density at the Sun and comparing with the in situ dust detected with Ulysses and Galileo, we can, in principle, infer the interstellar grain content, at least for the larger grains. In Fig. 5, as an example, we show the grain size distribution that an MRN (Mathis et al., 1977) type size distribution in the ISM would produce at the Sun under the assumption of a focusing polarity in the SWMF. Here the size distribution has the MRN power law in size, $d n / d a \propto a_{\text {grain }}^{-3.5}$ (but we show the differential mass per logarithmic grain mass distribution), though the grain size limits extend from 0.01 to $1.0 \mu \mathrm{m}$ instead of the size limits used to fit interstellar extinction which go from $\sim 0.005 \mu \mathrm{m}$ to $0.25 \mu \mathrm{m}$. A gas-to-dust mass ratio of 150 is assumed to set the overall normalization of the distribution. It's clear from the figure that this "shifted MRN" distribution overpredicts the dust density at the Sun. As mentioned above, however, the assumption of a single polarity for the SWMF over the entire course of a grain trajectory is not realistic. Future modeling will need to take the time varying SWMF into account.

\section{Life Cycle of Local Interstellar Dust Grains}

Variations in the relative abundances of $\mathrm{Mg}^{+}$and $\mathrm{Fe}^{+}$ in the gas by a factor of 30 between different CLIC velocity components (Fig. 2) indicates an inhomogeneous grain population. Either multiple grain sources or the non-uniform destruction of the initial grain population may account for these differences. The kinematical coupling between the gas and dust suggests that the local dust is far removed from the original source in active stars and supernova, or massive molecular clouds. Rather, it appears that there have been different levels of grain destruction in the CLIC, and that possibly grain destruction is ongoing. The very nature of the dispersion of velocities in the CLIC $\left( \pm 4.6 \mathrm{~km} \mathrm{~s}^{-1}\right.$ about the bulk flow vector, Frisch et al. (2011), Fig. 1) suggests that local dust grains may not have been uniformly shocked. In the Fe core-silicate mantle model, shock processing that removes only the mantle, such as shocks with velocities below $100 \mathrm{~km} \mathrm{~s}^{-1}$, will preferentially return $\mathrm{C}, \mathrm{Si}$ and $\mathrm{Mg}$ to the gas phase as compared to Fe by thermal sputtering (Fig. 6). Iron oxides may survive shocks in the cores of grains that have protective mantles of a lower density material.

The efficiency of grain destruction by shocks depends on the properties of the medium into which it propagates. If the pre-shock gas were inhomogeneous, the efficiencies of grain destruction will have varied. For instance, increasing the cloud density by a factor of ten raises the grain destruction rate by a factor of $\sim 1.5$, while increasing the interstellar magnetic field strength from $1 \mu \mathrm{G}$ to $3 \mu \mathrm{G}$ reduces the grain destruction rate by factor of $\sim 2$.

\subsection{Dust destruction in the CLIC}

Our photoionization models for the LIC (Slavin and Frisch, 2008) use the observed LIC column densities toward $\epsilon$ CMa to set the gas phase elemental abundances. We then compare the abundances derived this way with a set of solar abundances to indicate the amount of depletion into grains. As can be seen in Fig. 3, we find modest depletions of the refractory elements that make up silicate grains (Fe, $\mathrm{Mg}, \mathrm{Si}$, 

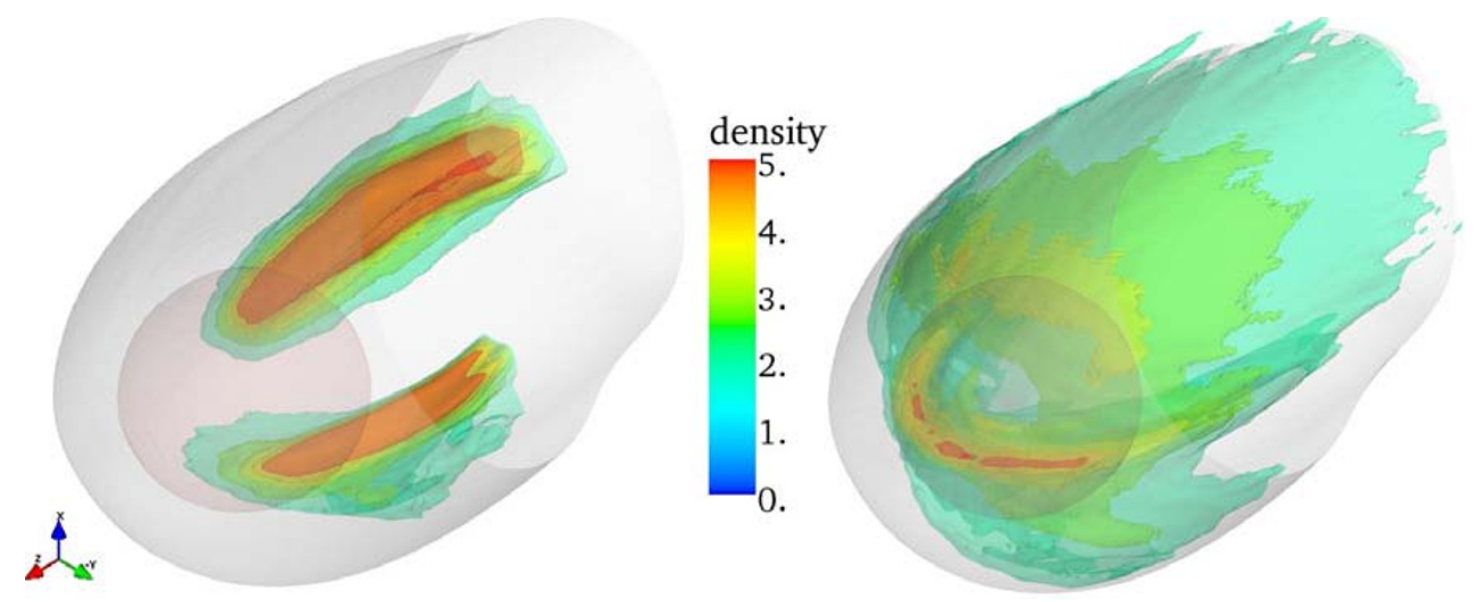

Fig. 4. Dust density distribution for $0.18 \mu \mathrm{m}$ grains in the heliosphere rendered as the 3-D surfaces corresponding to factors of $2,2.75,3.5,4.25$ and 5 enhancement over the ambient density. The de-focusing SWMF case is shown on the left and the focusing SWMF polarity case is shown on the right. The focusing SWMF leads to enhanced density in a crescent shaped region near the ecliptic plane while the de-focusing field leads to concentration of the dust at the north and south ecliptic poles. The inner, nearly spherical surface is the solar wind termination shock. Note: the interstellar inflow comes from the lower left and flows toward the upper right. (Figure from Slavin et al., 2012).

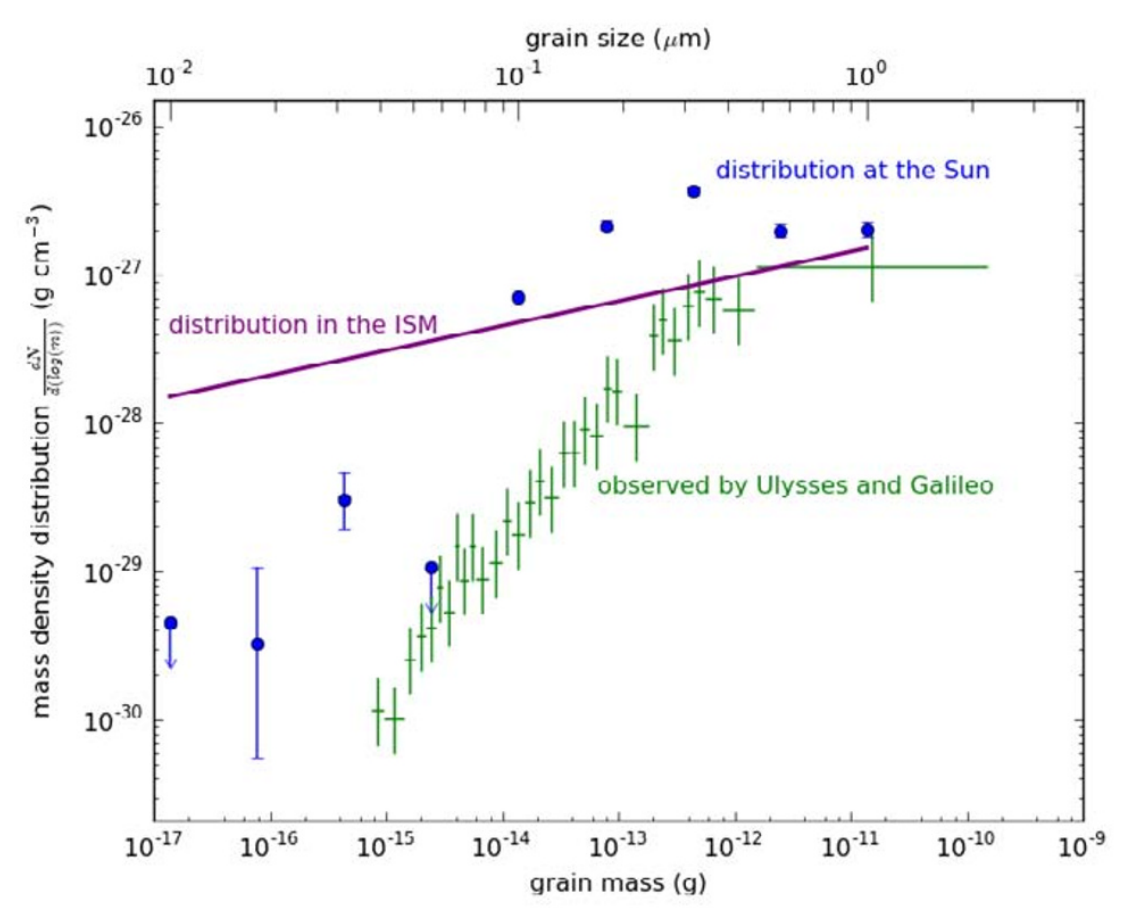

Fig. 5. Grain mass distribution for interstellar grains in the heliosphere observed by the Ulysses and Galileo spacecrafts (updated, H. Krueger private communication). As an example, the purple line shows the MRN power law size distribution (Mathis et al., 1977) but with higher upper and lower size cutoffs and using a gas-to-dust mass ratio of 150. The blue points show the grain mass distribution that results in our model (focusing SWMF). This model clearly overpredicts the density of large grains, but is promising for linking the observed grain size distribution with the initial one in the Local Interstellar Cloud.

and $\mathrm{O}$ ) and no depletion of $\mathrm{C}$. This low level of depletion in the LIC can be interpreted as evidence of partial destruction of grains by a fast shock sometime in the past.

In relatively slow shocks, $v_{\text {shock }} \sim 50-100 \mathrm{~km} \mathrm{~s}^{-1}$, the grain destruction is primarily via grain-grain collisions caused by the betatron acceleration of the grains in the postshock cooling region. This sort of destruction tends to erode any mantle the grains might have and shift the grain size distribution towards smaller grains. In faster shocks, thermal and non-thermal sputtering preferentially destroy smaller grains by removing the same thickness of grain material re- gardless of grain size. In Fig. 6, left, we show the resulting depletions after shock passage for a core-mantle grain model wherein the core is silicate and mantle is pyroxene (from Frisch et al., 1999). The effects of shock passage depend on, in addition to assumptions about grain composition and structure, the gas density and magnetic field strength.

The abundances derived for the LIC present a challenge for these dust destruction models since the prediction is that carbonaceous dust will be less destroyed than silicate dust for shock velocities over $150 \mathrm{~km} \mathrm{~s}^{-1}$. The depletions we 
(a)

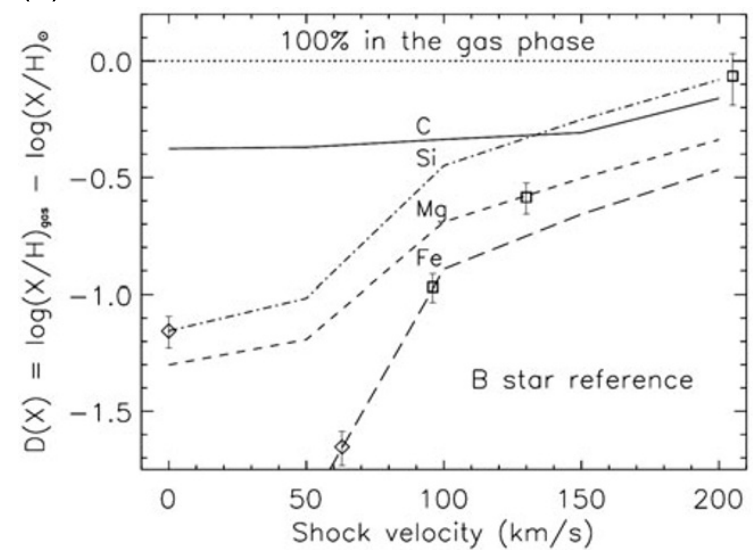

(b)

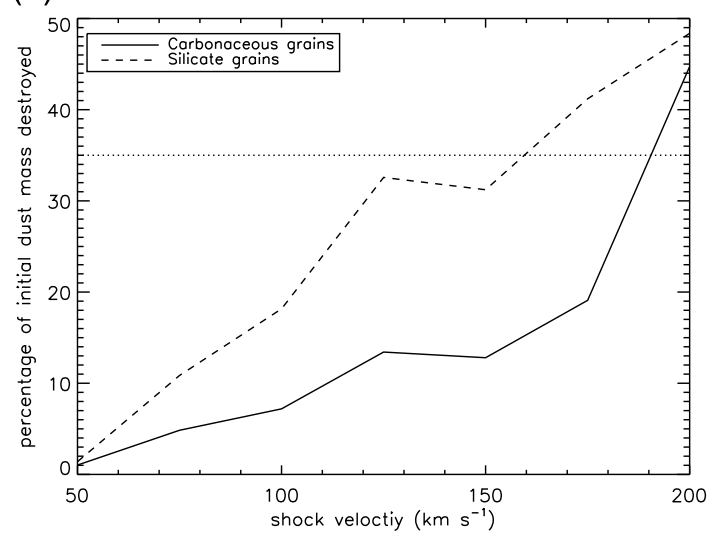

Fig. 6. Left: Post-shock depletion vs. shock velocity for core-mantle grain model where core is assumed to be silicate (olivine) and mantle is pyroxene (from Frisch et al., 1999). Right: Percentage of initial total grain mass lost because of dust destruction in the shock vs. shock speed. To get the Si gas phase abundance determined for the LIC we need $\lesssim 35 \%$ destruction of silicate dust which is consistent with $v_{\text {shock }} \leq 150 \mathrm{~km} \mathrm{~s}{ }^{-1}$. Such shocks should destroy less than $15 \%$ of the carbonaceous dust, however, in contradiction with the large gas phase abundance of carbon found in many studies (also see Section 3.2).

find for silicate grain elements imply that $\sim 35 \%$ of the grain mass has been destroyed as could occur if a shock of $\sim 150$ $\mathrm{km} \mathrm{s}^{-1}$ has passed through the cloud, but as can be seen from Fig. 6, right, the shock destruction models indicate that $<15 \%$ of the carbonaceous grains should have been destroyed by such a shock. A similar pattern of high carbon dust destruction and modest silicate dust destruction is inferred for lines of sight toward high and intermediate velocity clouds (Welty et al., 2002). These results prompted Jones and Nuth (2011) to propose that instead of graphite as the model for carbonaceous grains, hydrogenated amorphous carbon might be closer to their structure. Calculations they did for these grains indicate that they are much easier to destroy in shocks, consistent with the above results.

\subsection{Source of very large interstellar grains}

Large and small interstellar dust grains in the LIC may originate in different regions of the CLIC. The gyroradius for $1 \mu \mathrm{m}$ silicate grain is $\sim 1 \mathrm{pc}$ for a grain with a density of $3.3 \mathrm{gr} \mathrm{cm}^{-3}$, charge of 200 , and traveling $23 \mathrm{~km} \mathrm{~s}^{-1}$ through a $3 \mu \mathrm{G}$ magnetic field. Small, radius $a \sim 0.1 \mu \mathrm{m}$, grains originate locally. Grains that are $1 \mu \mathrm{m}$ and larger may originate in more distant regions of the CLIC. The presence of large ISDGs in the ISM, radius $a>1 \mu \mathrm{m}$, if widespread, would violate abundance constraints set by interstellar extinction curves. This suggests that they trace a different ISM reservoir than the small grains (Draine, 2009). The large gyroradius of the large grains allows for them to be decoupled from the small grains so that the grain population may not be uniformly shocked.

The large grains (radius $a>1 \mu \mathrm{m}$ ) found by in situ measurements of interstellar dust suggests that molecular cloud grains are mixed into the local ISM. Infrared observations of dense molecular clouds require micron-sized ISDGs to reproduce the "coreshine" emission. Coreshine is produced when dusty clouds scatter background $8 \mu \mathrm{m}$ radiation that is spatially coincident with the cloud cores (Pagani et al., 2010). The only known dense dust cloud near the Sun is a thin filamentary-like cold $(30 \mathrm{~K})$ feature towards Leo. The distance of this cloud has been constrained by absorption lines in nearby stars to be within 11-24 pc (Frisch et al., 2011; Peek et al., 2011). Densities in the Leo cloud are $N\left(\mathrm{H}^{\mathrm{o}}\right) \sim 10^{19}-10^{20} \mathrm{~cm}^{-2}$, and $n \sim 320 \mathrm{~cm}^{-3}$. The Leo cloud velocity is consistent with the CLIC bulk velocity, so either this cloud or other similar tiny dense local clouds (e.g. Haud, 2010) may explain the large dust grains observed inside of the heliosphere.

\section{Summary}

Local interstellar dust shows the characteristics of dust in low density clouds, with high gas phase abundances of refractory elements that have been processed by $\sim 150 \mathrm{~km} \mathrm{~s}^{-1}$ shocks. Grains consist of silicates, primarily olivine, and an additional Fe-rich grain component, possibly of complex oxides. Observations of interstellar oxygen both inside and outside of the heliosphere suggest high depletions that indicate an additional carrier of the oxygen such as either organic refractory particles or oxides. The proximity of the Sun to the Loop I superbubble suggests that grain destruction may have occurred in shocks associated with that superbubble.

Large $>1 \mu \mathrm{m}$ interstellar dust grains have been detected in the heliosphere, indicating either that some grains escaped destruction in interstellar shocks, or that this dust has decoupled from a nearby reservoir of large grains, or both. The observed deficit of small grains, $a<0.1 \mu \mathrm{m}$, in the in situ grain sample indicates that the smallest grains are excluded from heliospheric plasma because of large charge-to-mass ratios. Models of this exclusion indicates that plumes of $\sim 0.18 \mu \mathrm{m}$ dust flow around the heliosphere, with the exact configuration of the plumes dependent on the solar wind magnetic field polarity and therefore on the solar cycle phase. These diverse techniques for studying local interstellar dust provide an intriguing and still incomplete picture of the properties of dust in low density regions of the galaxy.

Acknowledgments. This work has been supported by the Inter- 
stellar Boundary Explorer mission as a part of NASA's Explorer Program, and by NASA grant NNX08AJ33G to the University of Chicago. The authors would like to thank an anonymous referee for emphasizing that iron oxides help to explain the observed characteristics of the local depletion patterns.

\section{References}

Asplund, M., N. Grevesse, and A. J. Sauval, The Solar Chemical Composition, in ASP Conf. Ser. 336: Cosmic Abundances as Records of Stellar Evolution and Nucleosynthesis, pages 25-28, 2005.

Asplund, M., N. Grevesse, A. J. Sauval, and P. Scott, The chemical composition of the Sun, Ann. Rev. Astron. Astrophys., 47, 481-522, 2009.

Bochsler, P., L. Petersen, E. Möbius, N. A. Schwadron, P. Wurz, J. A. Scheer, S. A. Fuselier, D. J. McComas, M. Bzowski, and P. C. Frisch, Estimation of the neon/oxygen abundance ratio at the heliospheric termination shock and in the local interstellar medium from IBEX observations, Astrophys. J. Suppl., 198, 13, 2012.

Cartledge, S. I. B., J. T. Lauroesch, D. M. Meyer, and U. J. Sofia, The homogeneity of interstellar elemental abundances in the galactic disk, Astrophys. J., 641, 327-346, 2006.

Draine, B. T., Perspectives on interstellar dust inside and outside of the heliosphere, Space Sci. Rev., 143, 333-345, 2009.

Fitzgerald, M. P., The distribution of interstellar reddening material, Astron. J., 73, 983, 1968.

Frisch, P. C., LISM Structure-Fragmented superbubble shell?, Space Sci. Rev., 78, 213-222, 1996.

Frisch, P. C. and J. D. Slavin, The chemical composition and gas-to-dust mass ratio of nearby interstellar matter, Astrophys. J., 594, 844-858, 2003.

Frisch, P. C., J. M. Dorschner, J. Geiss, J. M. Greenberg, E. Grün, M. Landgraf, P. Hoppe, A. P. Jones, W. Krätschmer, T. J. Linde, G. E. Morfill, W. Reach, J. D. Slavin, J. Svestka, A. N. Witt, and G. P. Zank, Dust in the local interstellar wind, Astrophys. J., 525, 492-516, 1999.

Frisch, P. C., M. Bzowski, E. Grün, V. Izmodenov, H. Krüger, J. L. Linsky, D. J. McComas, E. Möbius, S. Redfield, N. Schwadron, R. R. Shelton, J. D. Slavin, and B. E. Wood, The galactic environment of the Sun: Interstellar material inside and outside of the heliosphere, Space Sci. Rev., 146, 235-273, 2009.

Frisch, P. C., B. Andersson, A. Berdyugin, H. O. Funsten, M. Magalhaes, D. J. McComas, V. Piirola, N. A. Schwadron, J. D. Slavin, and S. J. Wiktorowicz, Comparisons of the interstellar magnetic field directions obtained from the IBEX ribbon and interstellar polarizations, Astrophys. J., 724, 1473-1479, 2010.

Frisch, P. C., S. Redfield, and J. D. Slavin, The interstellar medium surrounding the Sun, Ann. Rev. Astron. Astrophys., 49, 237-279, 2011.

Frisch, P. C., B. Andersson, A. Berdyugin, H. O. Funsten, M. Magalhaes, D. J. McComas, W. DeMajistre, V. Piirola, N. A. Schwadron, J. D. Slavin, and S. J. Wiktorowicz, Comparisons of the interstellar magnetic field directions obtained from the IBEX ribbon and interstellar polarizations. II, Astrophys. J., 760, 106-124, 2012.

Haud, U., Gaussian decomposition of HI surveys. V. Search for very cold clouds, Astron. Astrophys., 514(A27), 2010.

Howk, J. C., B. D. Savage, and D. Fabian, Abundances and physical conditions in the warm neutral medium toward $\mu$ Columbae, Astrophys. J., 525, 253-293, 1999.

Jenkins, E. B., A unified representation of gas-phase element depletions in the interstellar medium, Astrophys. J., 700, 1299-1348, 2009.

Jones, A. P., Iron or iron oxide grains in the interstellar medium?, Mon. Not. R. Astron. Soc., 245, 331-334, 1990.

Jones, A. P. and J. A. Nuth, Dust destruction in the ISM: a re-evaluation of dust lifetimes, Astron. Astrophys., 530, A44, 2011.

Juett, A. M., N. S. Schulz, D. Chakrabarty, and T. W. Gorczyca, Highresolution X-ray spectroscopy of the interstellar medium. II. Neon and iron absorption edges, Astrophys. J., 648, 1066-1078, 2006.

Kimura, H., I. Mann, and E. K. Jessberger, Composition, structure, and size distribution of dust in the local interstellar cloud, Astrophys. J., 583, 314-321, 2003a.

Kimura, H., I. Mann, and E. K. Jessberger, Elemental abundances and mass densities of dust and gas in the local interstellar cloud, Astrophys. J., 582, 846-858, 2003b.

Krüger, H., V. Dikarev, B. Anweiler, S. F. Dermott, A. L. Graps, E. Grün, B. A. Gustafson, D. P. Hamilton, M. S. Hanner, M. Horányi, J. Kissel, D. Linkert, G. Linkert, I. Mann, J. A. M. McDonnell, G. E. Morfill,
C. Polanskey, G. Schwehm, and R. Srama, Three years of Ulysses dust data: 2005 to 2007, Planet. Space Sci., 58, 951-964, 2010.

Leske, R. A., Anomalous cosmic ray composition from ACE, in AIP Conf. Proc. 516: 26th International Cosmic Ray Conference, ICRC XXVI, edited by B. L. Dingus, D. B. Kieda, and M. H. Salamon, page 274, 2000

Mann, I., Interstellar dust in the solar system, Ann. Rev. Astron. Astrophys., 48, 173-203, 2009.

Mathis, J. S., W. Rumpl, and K. H. Nordsieck, The size distribution of interstellar grains, Astrophys. J., 217, 425-433, 1977.

McComas, D. J., F. Allegrini, P. Bochsler, M. Bzowski, E. R. Christian, G. B. Crew, R. DeMajistre, H. Fahr, H. Fichtner, P. C. Frisch, H. O. Funsten, S. A. Fuselier, G. Gloeckler, M. Gruntman, J. Heerikhuisen, V. Izmodenov, P. Janzen, P. Knappenberger, S. Krimigis, H. Kucharek, M. Lee, G. Livadiotis, S. Livi, R. J. MacDowall, D. Mitchell, E. Möbius, T. Moore, N. V. Pogorelov, D. Reisenfeld, E. Roelof, L. Saul, N. A Schwadron, P. W. Valek, R. Vanderspek, P. Wurz, and G. P. Zank, Global observations of the interstellar interaction from the Interstellar Boundary Explorer (IBEX), Science, 326, 959, 2009.

McComas, D. J., D. Alexashov, M. Bzowski, H. Fahr, J. Heerikhuisen, V. Izmodenov, M. A. Lee, E. Möbius, N. V. Pogorelov, N. A. Schwadron, and G. P. Zank, The heliosphere's interstellar interaction: No bow shock, Science, 336, 1291-1293, 2012.

Pagani, L., J. Steinacker, A. Bacmann, A. Stutz, and T. Henning, The ubiquity of micrometer-sized dust grains in the dense interstellar medium, Science, 329, 1622-1624, 2010.

Peek, J. E. G., C. Heiles, K. M. G. Peek, D. M. Meyer, and J. T. Lauroesch, The local leo cold cloud and new limits on a local hot bubble, Astrophys. J., 735, 129-141, 2011.

Redfield, S. and J. L. Linsky, The structure of the local interstellar medium. IV. dynamics, morphology, physical properties, and implications of cloud-cloud interactions, Astrophys. J., 673, 283-314, 2008.

Redfield, S. and J. L. Linsky, The structure of the local interstellar medium. I. High-resolution observations of Fe II, Mg II, and Ca II toward stars within 100 parsecs, Astrophys. J. Suppl., 139, 439-465, 2002.

Redfield, S. and J. L. Linsky, The structure of the local interstellar medium. II. Observations of D I, C II, N I, O I, Al II, and Si II toward stars within 100 parsecs, Astrophys. J., 602, 776-802, 2004.

Slavin, J. D. and P. C. Frisch, The boundary conditions of the heliosphere: photoionization models constrained by interstellar and in situ data, Astron. Astrophys., 491, 53-68, 2008.

Slavin, J. D., P. C. Frisch, J. Heerikhuisen, N. V. Pogorelov, H.-R. Mueller, W. T. Reach, G. P. Zank, B. Dasgupta, and K. Avinash, Exclusion of tiny interstellar dust grains from the heliosphere, Twelfth Int. Sol. Wind Conf., 1216, 497-501, 2010.

Slavin, J. D., P. C. Frisch, W. T. Reach, J. Heerikhuisen, N. V. Pogorelov, H. R. Mueller, and G. P. Zank, Emission from interstellar dust in the heliosphere, in American Astronomical Society Meeting Abstracts \#218, page No. 129.25, 2011.

Slavin, J. D., P. C. Frisch, J. Heerikhuisen, N. V. Pogorelov, W. T. Reach, H.-R. Müller, G. Zank, and K. Avinash, Trajectories and distribution of interstellar dust grains in the heliosphere, Astrophys. J., 60, 46-61, 2012.

Sofia, U. J., K. D. Gordon, G. C. Clayton, K. Misselt, M. J. Wolff, N. L. J Cox, and P. Ehrenfreund, Probing the dust responsible for small magellanic cloud extinction, Astrophys. J., 636, 753-764, 2006.

Welty, D. E., J. T. Lauroesch, J. C. Blades, L. M. Hobbs, and D. G. York, Unusual depletions toward the SMC star SK 155-differences in dust composition in the SMC interstellar medium?, Astrophys. J. Lett., 554, L75-L79, 2001.

Welty, D. E., E. B. Jenkins, J. C. Raymond, C. Mallouris, and D. G. York, Intermediate- and high-velocity ionized gas toward $\zeta$ Orionis, Astrophys. J., 579, 304-326, 2002.

Whittet, D. C. B., Oxygen Depletion in the interstellar medium: Implications for grain models and the distribution of elemental oxygen, Astrophys. J., 710, 1009-1016, 2010.

Wolleben, M., A new model for the Loop I (North Polar Spur) region, Astrophys. J., 664, 349-356, 2007.

Zubko, V., E. Dwek, and R. G. Arendt, Interstellar dust models consistent with extinction, emission, and abundance constraints, Astrophys. J. Suppl., 152, 211-249, 2004.

P. C. Frisch (e-mail: frisch@oddjob.uchicago.edu) and J. D. Slavin 